\title{
Protecting Autonomy of Rohingya Women in Sexual and Reproductive Health Interventions
}

\author{
Haaris Siddiqi*
}

\section{ABSTRACT}

Rohingya women face challenges that ought to be acknowledged and addressed to ensure that when they seek health care, they can act autonomously and decide freely among available options. Self-determination theory offers valuable insight into supporting these women within their unique situations.

Keywords: Rohingya Women, Autonomy, Reproductive Health, Paternalism, Informed Consent, Muslim

\section{INTRODUCTION}

In August of 2017, military and paramilitary forces in Myanmar began purging the Rohingya Muslim population from the country, motivated by anti-Muslim prejudice of the Buddhist political and social majority. Mass murder, property destruction, kidnapping, torture, and sexual violence still affect Rohingya communities. As a result, more than a million individuals have fled Myanmar. ${ }^{1}$ As of February 2021 , approximately 880,000 Rohingya Muslims have taken refuge in Cox's Bazar, Bangladesh, the site of the largest refugee camps in the world. ${ }^{2}$ The public health focus in these camps is on treatment of physical ailments and infectious diseases. ${ }^{3}$ While women of reproductive age and adolescent girls experience the highest level of violence among Rohingya communities in both Myanmar and Bangladesh, they have consistently lacked access to sufficient sexual and reproductive care.

In 1994, the Women's Commission for Refugee Women and Children exposed issues surrounding the sexual and reproductive health of displaced populations and propelled the recognition of SRH as a human right. ${ }^{4}$ Human rights interventionists and public health officials have made progress in the integration of sexual and reproductive health education, facilities, and resources into refugee camps in Cox's Bazar. This includes the introduction of menstrual cleanliness facilities and educational conversations. However, Rohingya women and male cultural leaders, or gatekeepers, remain reluctant to accept these resources and education. ${ }^{5}$ The prevalence of gender-based violence against women and restrictive policies enforced by

\footnotetext{
* Haaris Siddiqi
}

(C) 2021 Haaris Siddiqi. This is an open access article distributed under the terms of the Creative Commons Attribution License, which permits unrestricted use, distribution, and reproduction, provided the original author and source are credited. 
the Bangladesh government heighten the barriers to the effective introduction of sexual and reproductive health resources and services. ${ }^{6}$

A wealth of literature has pushed for the extension of clinical duties of beneficence and non-maleficence in the diagnosis and treatment of refugee and asylum-seeking communities. ${ }^{7}$ Additionally, extensive research on Rohingya refugee communities has searched for ways to work around the complex social history and to accommodate power structures by integrating gatekeepers into SRH discussions. ${ }^{8}$ However, as interventions have sought to overcome cultural and religious barriers, they have largely overlooked the protection of autonomy of sexual and reproductive health patients in Cox's Bazar. This paper argues two points. First, attempts at improving outcomes in Cox's Bazar ought to lead to Rohingya women's autonomy and self-determination, both in mitigating control of male leaders over sexual and reproductive decisions and in ensuring the understanding and informed consent between patients and providers. Second, policy decisions ought to ensure post-treatment comprehensive care to shield Rohingya women from retribution by male community members. Self-determination theory offers guidance for state leaders and healthcare providers in pursuing these goals.

\section{Barriers to Sexual and Reproductive Health Services for Rohingya Women}

As part of its anti-Muslim narrative, the Buddhist majority has painted Rohingya women as hyperreproductive. False narratives "of a Rohingya plan to spread Islam by driving demographic shifts" and accusations against Rohingya women for having "unusually large families" have motivated violent behavior and discriminatory regulations against Rohingya communities. ${ }^{9}$ In reality, demographic data shows that "the Rohingya population has remained stable at 4\% since 1980." ${ }^{10}$ In 2013, the government of Myanmar imposed regulations on Rohingya families in the Rakhine state, the region with the highest population of Rohingya Muslims, enforcing a two-child limit and requiring that Rohingya women obtain government authorization to marry and take a pregnancy test before receiving such permission. The majority has also subjected Rohingya females to acts of sexual violence to ostracize them and "dilute" Rohingya identity. ${ }^{11}$

As a result, Rohingya women in Cox's Bazar experience unique illnesses and vulnerabilities requiring imminent treatment. Due to national policies in Bangladesh, "Rohingya [women] cannot receive HIV/AIDS testing and treatment in camps; birth control implants delivered by midwives; and comprehensive abortion care." ${ }^{12}$ Additionally, in accordance with patriarchal Rohingya community structure, male gatekeepers hold high authority over sexual and reproductive decisions of women, evidenced by the persistence of genderbased violence within refugee camps and traditional practices such as the marriage of minor girls to older Rohingya men. ${ }^{13}$ Surveys of community members reveal that cultural and religious stigma against sexual and reproductive health care exists among these male gatekeepers as well as Rohingya women. ${ }^{14}$ Due to their cultural and political position, Rohingya women are subject to unique power relations. This paper analyzes the ethical dilemmas that arise from two of those power relations: Rohingya women's relationships with male gatekeepers and their relationships with interventionist healthcare providers.

II. Ethics of Including Male Community Members in Decisions Affecting Women's Healthcare Autonomy

A November 2019 survey of Rohingya women in Cox's Bazar that had married or given birth within the past two years found that "around one half of the female Rohingya refugees do not use contraceptives, mainly because of their husbands' disapproval and their religious beliefs." ${ }^{15}$ There are widespread misconceptions such as the belief that Islam does not permit the use of contraceptives. ${ }^{16}$ The existence of such misconceptions and the power husbands and male leaders hold over the delivery of treatment creates dilemmas for healthcare practitioners in conforming to ethical principles of care. 


\section{Beneficence in Providing Care to Refugees}

While public health scholars and government officials hold divided opinions on the level of treatment required to fulfill refugees' right to sexual and reproductive health care, most support enough care to ensure physical and psychological well-being. ${ }^{17}$ Beneficence requires that healthcare providers and states "protect the rights of others[,] prevent harm from occurring to others[, and] remove conditions that will cause harm to others." ${ }^{18}$ Under the principle of beneficence, there is a duty to provide sexual and reproductive treatment to Rohingya women in Cox's Bazar that is comparable to that received by citizens of the host state. In addition, the ethical principle of nonmaleficence may call for the creation of specialized care facilities for refugee communities, because a lack of response to refugees' vulnerability and psychological trauma has the potential to generate additional harm. ${ }^{19}$

In response to gendered power relations among the Rohingya community, husbands and male leaders are included in decisions surrounding maternal health and sexual and reproductive care for women. For example, healthcare professionals "have been found to impose conditions on SRH [sexual reproductive health] care that are not stated in the national... [menstrual regulation] guidelines, such as having a husband's permission." ${ }^{20}$ The refugee healthcare community could do more to mitigate the potential of retribution taken by male community members against women that accept care by dispelling common misconceptions and precluding male community members from influencing female reproductive choices. ${ }^{21}$ However, some current practices allow the infiltration of male community leaders and husbands into the diagnosis, decision-making, and treatment spaces. Deferring decisions to male leaders for the sake of expediency risks conditioning women's access to care on male buy-in and diminishes Rohingya women's autonomy over their sexual and reproductive health.

\section{Male Influence and Female Autonomy}

Ensuring patients control their own treatment decisions is an essential component of the ethical obligation of healthcare professionals to respect patients' autonomy. While patients can exercise their autonomy to accept the direction of the community, their autonomy is undermined when "external sources or internal states... rob [such persons]... of self-directedness." 22 Sexual and reproductive health research on Rohingya women revealed that the presence of male family members during conversations "made female respondents uncomfortable to speak openly about their SRH [sexual and reproductive health]related experiences." ${ }^{23}$ The same study found that when male family members were absent, Rohingya women were more transparent and willing to discuss such topics. ${ }^{24}$ These findings indicate that the mere presence of male family members exerts control over Rohingya women in conversations with practitioners. Male involvement also stalls conversations between providers and Rohingya women which may harm the achievement of understanding and informed consent in diagnosis and treatment spaces. ${ }^{25}$ Women do have the option of bringing their male community leaders and family members into sexual health discussions. Yet healthcare providers ought to monitor patients individually and avoid programmatic decision making regarding male involvement in the treatment space. While it is the ethical imperative of health interventionists and the state of Bangladesh to fulfill the duties of care required by the principles of beneficence and non-maleficence, the sole prioritization of expanding sexual and reproductive health care in Cox's Bazar risks ignoring autonomy.

\section{Ethics of Paternalism in Provide-Patient Relations}

Rohingya women's negative beliefs about contraceptives, such as the belief that they cause irreversible sterilization, are the second largest factor inhibiting their use. ${ }^{26}$ To an extent, the Rohingya are justified in 
their skepticism. Prior to the 1990's, Bangladesh used nonconsensual sterilization as a mechanism of population control to attain access to international aid. Though the international conversation surrounding reproduction shifted its focus towards reproductive rights following the 1994 UN International Conference on Population and Development, delivery of reproductive care in the global South is frequently characterized by lack of transparency and insufficient patient understanding of the risks and consequences of treatment.

Additionally, women's lack of control impacts follow-up care and long-term contraception. For example, when women seek the removal of implantable contraceptives, healthcare professionals often refuse to perform the requisite operation. ${ }^{27}$ Patients must understand the risks of treatment in their own culture and circumstances where societal views, misconceptions, or fears may influence healthcare practices. Healthcare providers need to recognize the coercive potential they hold in their relations with patients and guard against breaches of patient autonomy in the delivery of treatment. In accordance with the principle of beneficence, healthcare providers treating refugees or individuals seeking asylum ought to abide by the same fiduciary responsibilities they hold toward citizens of the host state. ${ }^{28}$ When patients show hesitancy or refusal toward treatment, healthcare providers ought to avoid achieving treatment by paternalistic practice such as "deception, lying, manipulation of information, nondisclosure of information, or coercion." ${ }^{29}$ Although well-intentioned, this practice undermines the providers' obligation to respect patients' autonomy. ${ }^{30}$ The hesitancy of Rohingya women to accept some sexual or reproductive health care does not justify intentional lack of transparency, even when that treatment furthers their best health interests. However, paternalistic actions may be permissible and justified during medical emergencies. ${ }^{31}$

\section{Informed Consent}

Respecting Rohingya women's autonomy also places affirmative duties on healthcare providers to satisfy understanding and informed consent. However, language barriers and healthcare providers' misconceptions about Rohingya religion and culture impede the achievement of these core conditions of autonomy for Rohingya women. ${ }^{32}$ In an interview, a paramedic in Cox's Bazar described the types of conversations healthcare providers have with Rohingya women in convincing them to accept menstrual regulation treatment, a method to ensure that someone is not pregnant after a missed period: "We tell them [menstrual regulation] is not a sin... If you have another baby now, you will get bad impact on your health. You cannot give your children enough care. So, take MR [menstrual regulation] and care for your family." ${ }^{33}$ This message, like others conveyed to Rohingya women in counseling settings, carries unvalidated assumptions regarding the beliefs, needs, and desires of clients without making a proper attempt to confirm the truth of those assumptions. Healthcare providers' lack of cultural competence and limited understanding of Bangladesh's national reproductive health policy complicates communication with Rohingya women. Additionally, the use of simple language, though recommended by the WHO's guideline on Bangladesh's policy, is inadequate to sufficiently convey the risks and benefits of menstrual regulation and other treatments to Rohingya women. ${ }^{34}$

For informed consent to be achieved, "the patient must have the capacity to be able to understand and assess the information given, communicate their choices and understand the consequences of their decision." 35 Healthcare providers must convey sufficient information regarding the risks, benefits, and alternatives of treatment as well as the risks and benefits of forgoing treatment. ${ }^{36}$ Sexual and reproductive health policies and practices must aim to simultaneously mitigate paternalism, promote voluntary and informed choice among Rohingya women, and foster cultural and political competency among healthcare providers. 


\section{Self-Determination Theory}

Self-determination theory is a psychological model that focuses on types of natural motivation and argues for the fulfillment of three conditions shown to enhance self-motivation and well-being: autonomy, competence, and relatedness. ${ }^{37}$ According to the theory, autonomy is "the perception of being the origin of one's own behavior and experiencing volition in action;" competence is "the feeling of being effective in producing desired outcomes and exercising one's capacities;" and, relatedness is "the feeling of being respected, understood, and cared for by others." 38 Bioethicists have applied self-determination theory to health care to align the promotion of patient autonomy with traditional goals of enhancing patient wellbeing. Studies on the satisfaction of these conditions in healthcare contexts indicate that their fulfillment promotes better health outcomes in patients. ${ }^{39}$ Like principlism, self-determination theory in Cox's Bazar could allow for increased autonomy while maximizing the well-being of Rohingya women and behaving with beneficence

Fostering self-determination requires that healthcare professionals provide patients with the opportunity and means of voicing their goals and concerns, convey all relevant information regarding treatment, and mitigate external sources of control where possible. ${ }^{40}$ In Cox's Bazar, health care organizations in the region and the international community can act to ensure women seeking health care are respected and able to act independently. A patient-centered care model would provide guidelines for the refugee setting. ${ }^{41}$ Providers can maximize autonomy by utilizing language services to give SRH patients the opportunity and means to voice their goals and concerns, disclose sufficient information about risks, benefits, and alternatives to each procedure, and give rationales for each potential decision rather than prescribe a decision. They can promote the feeling of competence among patients by expressly notifying them of the level of reversibility of each treatment, introducing measures for health improvement, and outlining patients' progress in their SRH health. Finally, they can promote relatedness by providing active listening cues and adopting an empathetic, rather than condescending, stance. ${ }^{42}$

Healthcare organizations ought to provide training to promote cultural competency and ensure that practitioners are well-versed on national regulations regarding sexual reproductive health care in Bangladesh to avoid the presumption of patients' desires and the addition of unnecessary barriers to care. Increased treatment options would make autonomy more valuable as women would have more care choices.

Given the historical deference to international organizations like the UN and World Bank, multilateral and organizational intervention would likely bolster the expansion of treatment options. International organizations and donors ought to work with the government of Bangladesh to offer post-treatment comprehensive care and protection of women who choose treatment against the wishes of male community members to avoid continued backlash and foster relatedness. ${ }^{43}$

\section{CONCLUSION}

Rohingya women in Cox's Bazar, Bangladesh face unique power relations that ought to be acknowledged and addressed to ensure that when they seek health care, they are able to act autonomously and decide freely among available options. While providers have duties under the principles of beneficence and nonmaleficence, patient well-being is hindered when these duties are used to trump the obligation to respect patient autonomy. Current approaches to achieving sexual and reproductive health risk the imposition of provider and communal control. Self-determination theory offers avenues for global organizations, 
Bangladesh, donors, and healthcare providers to protect Rohingya women's autonomous choices, while maximizing their well-being and minimizing harm.

DISCLAIMER: As a male educated and brought up in a Western setting, I acknowledge my limitations in judgement about Rohingya women's reproductive care. Their vulnerability and health risks can never be completely understood. To some extent, those limitations informed my theoretical approach and evaluation of Rohingya women SRH care. Self-determination theory places the patients' experiences and judgement at the center of decision-making. My most important contributions to the academic conversation surrounding Rohingya women are the identification of dilemmas where autonomy is at risk and advocating for self-determination.

\footnotetext{
${ }^{1}$ Hossain Mahbub, Abida Sultana, and Arindam Das, "Gender-based violence among Rohingya refugees in Bangladesh: a public health challenge," Indian Journal of Medical Ethics (June 2018):1-2, https://doi.org/10.20529/IJME.2018.045.
}

2 "UN teams assisting tens of thousands of refugees, after massive fire rips through camp in Bangladesh," United Nations, last modified March 23, 2021, https://news.un.org/en/story/2021/03/1088012\#: :text=The\%20Kutupalong\%20camp\%20network\%2C\%20which, (as\%20of\%20February\%202021).

${ }^{3}$ Hossain et al., "Gender-based violence," 1-2.

${ }^{4}$ Benjamin O. Black, Paul A, Bouanchaud, Jenine K. Bignall, Emma Simpson, Manish Gupta, "Reproductive health during conflict," The Obstetrician and Gynecologist 16, no. 3 (July 2014):153-160, https://doi.org/10.1111/tog.12114.

${ }^{5}$ Margaret L. Schmitt, Olivia R. Wood, David Clatworthy, Sabina Faiz Rashid, and Marni Sommer, "Innovative strategies for providing menstruation-supportive water, sanitation and hygiene (WASH) facilities: learning from refugee camps in Cox's bazar, Bangladesh," Conflict and Health Journal 15, no. 1 (Feb 2021):10, https://doi.org/10.1186/s13031-021-00346-9.

${ }^{6} \mathrm{~S}$ M Hasan ul-Bari, and Tarek Ahmed, "Ensuring sexual and reproductive health and rights of Rohingya women and girls," The Lancet 392, no. 10163:2439-2440, https://doi.org/10.1016/S0140-6736(18)32764-8.

7 Janet Cleveland, and Monica Ruiz-Casares, "Clinical assessment of asylum seekers: balancing human rights protection, patient well-being, and professional integrity," American Journal of Bioethics 13, no. 7 (July 2013):13-5, https://doi.org/10.1080/15265161.2013.794885.; Christine Straehle, "Asylum, Refuge, and Justice in Health," Hastings Center Report 49, no. 3 (May/June 2019):13-17, https://doi.org/10.1002/hast.1002.

${ }^{8}$ Hossain et al., "Gender-based violence," 1-2.; Schmitt et al., "Innovative strategies," 10.

${ }^{9}$ Audrey Schmelzer, Tom Oswald, Mike Vandergriff, and Kate Cheatham, "Violence Against the Rohingya a Gendered Perspective," Praxis: The Fletcher Journal of Human Security, last modified February 11, 2021, https://sites.tufts.edu/praxis/2021/02/11/violence-against-the-rohingya-a-gendered-perspective/.

${ }^{10}$ Schmelzer et al., "Violence Against."

${ }^{11}$ Schmelzer et al., "Violence Against."

${ }^{12}$ LiesI Schnabel, and Cindy Huang, "Removing Barriers and Closing Gaps: Improving Sexual and Reproductive Health and Rights for Rohingya Refugees and Host Communities," Center for Global Development: CGD Notes (June 
2019):6, https://www.cgdev.org/sites/default/files/removing-barriers-and-closing-gaps-improving-sexual-andreproductive-health-and-rights.pdf.

${ }^{13}$ Schnabel and Huang, "Removing Barriers," 4-9.; Andrea J. Melnikas, Sigma Ainul, lqbal Ehsan, Eashita Haque, and Sajeda Amin, "Child marriage practices among the Rohingya in Bangladesh," Conflict and Health Journal 14, no. 28 (May 2020), https://doi.org/10.1186/s13031-020-00274-0.

${ }^{14}$ Nuruzzaman Khan, Mofizul Islam, Mashiur Rahman, and Mostafizur Rahman, "Access to female contraceptives by Rohingya refugees, Bangladesh," Bull World Health Organ, 99, no.3 (March 2021):201-208, https://doi.org/10.2471/BLT.20.269779.

${ }^{15}$ Khan et al., "Access to," 201-208.

${ }^{16}$ Khan et al., “Access to," 201-208.

${ }^{17}$ Ramin Asgary, and Clyde L. Smith, "Ethical and professional considerations providing medical evaluation and care to refugee asylum seekers," American Journal of Bioethics 13, no. 7 (July 2013):3-12,

https://doi.org/10.1080/15265161.2013.794876.; Cleveland and Ruiz-Casares, "Clinical assessment," 13-5.;

Straehle, "Asylum," 13-17.

${ }^{18}$ Tom L. Beauchamp, and James Childress, Principles of Biomedical Ethics. Eighth Edition, (New York, NY: Oxford University Press, [1979] 2019), 219.

${ }^{19}$ Beauchamp and Childress, "Principles," 155.; Straehle, "Asylum," 15.

${ }^{20}$ Maria Persson, Elin C. Larsson, Noor Pappu Islam, Kristina Gemzell-Danielsson, and Marie Klingberg-Allvin, "A qualitative study on health care providers' experiences of providing comprehensive abortion care in Cox's Bazar, Bangladesh," Conflict and Health Journal 15, no. 1 (Jan 2021):3, https://doi.org/10.1186/s13031-021-00338-9.

${ }^{21}$ Rushdia Ahmed, Bachera Aktar, Nadia Farnaz, Pushpita Ray, Adbul Awal, Raafat Hassan, Sharid Bin Shafique, Md Tanvir Hasan, Zahidul Quayyum, Mohira Babaeva Jafarovna, Loulou Hassan Kobeissi, Khalid El Tahir, Balwinder Singh Chawla, and Sabina Faiz Rashid, "Challenges and strategies in conducting sexual and reproductive health research among Rohingya refugees in Cox's Bazar, Bangladesh," Conflict and Health Journal 14, no. 1 (Dec 2020):83, https://doi.org/10.1186/s13031-020-00329-2.; Khan et al., “Access to," 201-208.

${ }^{22}$ Beauchamp and Childress, Principles, 102.

${ }^{23}$ Ahmed et al., "Challenges and strategies," 6.

${ }^{24}$ Ahmed et al., "Challenges and strategies," 7.

${ }^{25}$ Beauchamp and Childress, Principles.

${ }^{26}$ Khan et al., "Access to," 201-208.

${ }^{27}$ Kalpana Wilson, "Towards a Radical Re-appropriation: Gender, Development and Neoliberal Feminism," Development and Change 46, no. 4 (July 2015):814-815, https://doi.org/10.1111/dech.12176.

${ }^{28}$ Asgary and Smith, "Ethical and professional," 3-12.

${ }^{29}$ Beauchamp and Childress, "Principles," 231.

${ }^{30}$ Beauchamp and Childress, "Principles," 231.

${ }^{31}$ Beauchamp and Childress, "Principles." 
32 Beauchamp and Childress, "Principles."

${ }^{33}$ Persson et al. "A qualitative study," 8.

${ }^{34}$ Persson et al. "A qualitative study."

${ }^{35}$ Christine S. Cocanour, "Informed consent-It's more than a signature on a piece of paper," American Journal of Surgery 214, no. 6 (Dec 2017):993, https://doi.org/10.1016/i.amjsurg.2017.09.015.

${ }^{36}$ Cocanour, "Informed consent," 993.

${ }^{37}$ Richard M. Ryan, and Edward L. Deci, "Self-determination theory and the facilitation of intrinsic motivation, social development, and well-being," American Psychologist 55, no. 1 (Jan 2000):68-78.

38 Johan Y.Y. Ng, Nikos Ntoumanis, Cecilie Thøgersen-Ntoumani, Edward L. Deci, Richard M. Ryan, Joan L. Duda, Geoffrey C. Williams, "Self-Determination Theory Applied to Health Contexts: A Meta-Analysis," Perspectives on Psychological Science 7, no. 4 (July 2021):325-340, https://doi.org/10.1177/1745691612447309.

${ }^{39} \mathrm{Ng}$ et al., "Self-Determination Theory."; Nikos Ntoumanis, Johan Y.Y. Ng, Andrew Prestwich, Eleanor Quested, Jennie E. Hancox, Cecilie Thøgersen-Ntoumani, Edward L. Deci, Richard M. Ryan, Chris Lonsdale \& Geoffrey C. Williams, "A meta-analysis of self-determination theory-informed intervention studies in the health domain: effects on motivation, health behavior, physical, and psychological health," Health Psychology Review 15, no. 2 (Feb 2020), https://doi.org/10.1080/17437199.2020.1718529.

${ }^{40}$ Leslie William Podlog, and William J. Brown, "Self-determination Theory: A Framework for Enhancing Patientcentered Care," The Journal for Nurse Practitioners 12, no. 8 (Sep 2016):e359-e362,

https://doi.org/10.1016/j.nurpra.2016.04.022.

${ }^{41}$ Podlog and Brown, "Self-determination Theory."

42 Podlog and Brown, "Self-determination Theory."

${ }^{43}$ Podlog and Brown, "Self-determination Theory." 